\title{
Derechos en el final de la vida: el rechazo de tratamientos médicos en los tribunales argentinos
}

\author{
The rights of the dying: the refusal of medical \\ treatments in Argentine courts
}

${ }^{1}$ Doctor en Ciencias Sociales. Investigador asistente, Consejo Nacional de Investigaciones Científicas y Técnicas (CONICET), Instituto de Investigaciones Gino Germani, Universidad de Buenos Aires (UBA). Buenos Aires, Argentina. $\square$ (iD)
RESUMEN El artículo aborda la judicialización de decisiones médicas en el final de la vida, en el marco del avance de la justicia en la regulación de la práctica médica y de un mayor reconocimiento de la autonomía de los pacientes. El artículo examina, desde una perspectiva sociológica, la jurisprudencia producida por tribunales argentinos entre los años 1975 y 2015 en torno al rechazo de tratamientos médicos en el final de la vida. Siguiendo un diseño metodológico cualitativo, se analizan 38 fallos, relevados a partir de descriptores en bases de datos jurisprudenciales. En primer lugar, se describen los casos judicializados, caracterizados por el protagonismo de las instituciones de salud como litigantes, el sesgo pro-tratamiento de los pedidos, y el alto porcentaje de demandas en ausencia de conflictos o en situaciones que no requerirían intervención judicial. En segundo lugar, se analizan los factores judiciales y extrajudiciales que inciden en la justiciabilidad del rechazo de tratamientos, como cambios normativos y procesos de politización de los reclamos.

PALABRAS CLAVES Negativa del Paciente al Tratamiento; Derecho a morir; Derecho a la Salud; Bioética; Argentina.

\begin{abstract}
This paper addresses the judicialization of end of life medical decisionmaking, as part of the advance of the justice system in the regulation of medical practice and the rise of recognition of patient autonomy. The article analyzes, from a sociological standpoint, legal decisions regarding treatment refusal at the end of life produced by the Argentine courts between 1975 and 2015. Based on a qualitative design, 38 sentences collected from jurisprudential databases using key terms were analyzed. First, judicialized cases during the period are described; these are characterized by a high proportion of claims presented by health institutions, a pro-treatment bias in the legal actions requested, and a high percentage of unnecessary litigation in the absence of conflicts or in situations that do not require court intervention. Second, legal and extralegal factors affecting the justiciability of decisions to refuse or withdraw medical treatments, such as changes in the law and processes of politicization of claims, are analyzed.
\end{abstract}

KEY WORDS Treatment Refusal; Right to Die; Right to Health; Bioethics; Argentina. 


\section{INTRODUCCIÓN}

En julio del año 2015, un fallo de la Corte Suprema de Justicia de la República Argentina autorizó la suspensión del soporte vital a un paciente que, desde hacía más de 20 años, se encontraba en estado vegetativo ${ }^{(1)}$. El fallo confirmó la constitucionalidad de la Ley 26742, más conocida como ley de "muerte digna", que garantiza el derecho de los pacientes con enfermedades terminales o irreversibles (o de los familiares en su nombre) a rechazar terapias o procedimientos médicos cuando estos sean desproporcionados a las perspectivas de mejoría o cuando prolonguen la agonía (2).

El citado fallo avivó el debate en el país acerca de los derechos de los pacientes en el final de la vida, así como del papel de la justicia en este tipo de decisiones ${ }^{(3,4,5)}$. En las últimas décadas, en un comienzo en países centrales y luego en diferentes partes del mundo, las decisiones médicas y las consecuencias de dichos actos, antes relegadas a la intimidad de la relación médico-paciente, comenzaron a llegar a tribunales judiciales ${ }^{(6,7)}$. El artículo examina la presencia en los tribunales argentinos de disputas en torno al rechazo de tratamientos médicos en el final de la vida, en el marco de procesos más amplios de judicialización de las relaciones sociales y de cambios en la práctica médica, marcados por un mayor reconocimiento de la autonomía de los pacientes respecto a las decisiones sobre la salud y el cuerpo, así como de la presencia de nuevos actores (como jueces y especialistas en bioética) en la regulación de estas decisiones.

El germen de la judicialización de la toma de decisiones médicas en el final de la vida se produce de la mano del avance de la medicalización y la tecnificación de la gestión de la muerte y el morir ${ }^{(8,9)}$. En la segunda mitad del siglo XX, los avances en la tecnología médica permitieron mantener el funcionamiento de órganos y funciones vitales por medios artificiales, habilitando la prolongación de la vida y la agonía ${ }^{(10,11)}$. En este marco, empiezan a tomar estado público casos controversiales en los que se pone en cuestión la autoridad de los profesionales médicos para tomar este tipo de decisiones.

El caso Quinlan marcó un hito en ese sentido, al poner por primera vez en el espacio público la discusión sobre los derechos de los pacientes a rechazar tratamientos de soporte vital. En 1976, la Suprema Corte de EE.UU. ordenó el retiro del respirador que mantenía con vida a Karen Quinlan, quien se encontraba en estado vegetativo, validando el pedido de los padres ante la negativa de los profesionales médicos a suspender la intervención. El caso constituyó uno de los primeros antecedentes de regulación de la práctica y las decisiones médicas en el final de la vida por parte de tribunales y jueces ${ }^{(7)}$.

El objetivo del artículo es describir y analizar la judicialización de los pedidos de rechazo de tratamientos en el final de la vida en Argentina, así como examinar los factores que promueven o dificultan el recurso a tribunales de justicia en este tipo de decisiones.

Los procesos de judicialización han recibido en los últimos años una importante atención por parte de cientistas sociales de América latina en torno a diferentes temáticas. Dichos procesos remiten a la ampliación de la esfera de intervención de cortes y tribunales y a la adopción, por parte de individuos y de grupos, de un discurso basado en derechos como vía para instrumentar demandas o reclamos ${ }^{(12,13,14,15,16,17,18,19)}$.

En el campo de la salud, la bibliografía sobre la judicialización se ha centrado en el análisis de disputas en torno al derecho a la salud, fundamentalmente a raíz del aumento de demandas por el acceso o la ampliación en la cobertura de medicamentos y servicios $(20,21,22,23,24,25,26,27)$. La judicialización de los actos médicos, como juicios por mala praxis y disputas sobre decisiones tanto al inicio como al final de la vida ${ }^{(28,29)}$ ha recibido, sin embargo, menor atención.

El artículo se enmarca en una investigación en curso que aborda la construcción de la gestión médica del final de la vida como problema público en Argentina, y examina el tratamiento de la temática en diferentes arenas (legislativa, jurídica y médica, entre 
otras). Interesa analizar aquí la presencia y las características de los debates en la arena jurídica a partir de un conjunto de fallos producidos por tribunales argentinos entre los años 1975 y 2015, como punto de partida para pensar temáticas relevantes para la sociología de la salud, como la judicialización de la práctica médica, los derechos de los pacientes, el papel de actores y discursos que regulan y condicionan el proceso de toma de decisiones, y la relación médico-paciente.

En el marco de este proyecto se analizó el proceso de politización de las demandas sobre los derechos en el final de la vida que culminó con el tratamiento legislativo y la sanción de la llamada ley de "muerte digna" en Argentina ${ }^{(3)}$, así como la participación de actores religiosos católicos en estos procesos $^{(4)}$. En un artículo previo se discutió el contexto y las implicancias sociales de la judicialización del rechazo de tratamientos desde una perspectiva sociohistórica, examinando los cambios en los lenguajes y argumentos desplegados en las disputas sobre decisiones médicas en el final de la vida ${ }^{(30)}$. En este artículo interesa describir la litigación sobre el rechazo de tratamientos en el final de la vida y, a modo de discusión, ponderar las condiciones para la judicialización de este tipo de conflictos.

\section{METODOLOGÍA}

La investigación en que se inscribe el artículo sigue una estrategia metodológica predominantemente cualitativa, basada en la recopilación y análisis de diferentes fuentes. Para la realización de este artículo se examinaron fallos judiciales producidos por tribunales argentinos (a nivel nacional, provincial y local) entre los años 1975 y 2015. El periodo analizado comprende desde los primeros casos de judicialización de la temática al reciente fallo del máximo tribunal de justicia del país que avala la constitucionalidad de la Ley $26742^{(1)}$.

La búsqueda de fallos se realizó en cinco bases de datos: una de las publicaciones de información jurídica más importante de Argentina, La Ley online (desde 1975); y las bases virtuales de la Corte Suprema de la Nación Argentina (desde 1994), de la Suprema Corte de la Provincia de Buenos Aires, del Tribunal Superior de Justicia de la Ciudad Autónoma de Buenos Aires (desde 1997) y el portal Infojus. La búsqueda en cada una de estas bases se realizó a partir de palabras claves. En virtud de los cambios en los casos judicializados se recurrió a una diversidad de descriptores ("muerte digna", "eutanasia", "objeción de conciencia", "bioética", entre otros). El corpus se completó con la búsqueda de fallos citados en otras sentencias o en notas periodísticas y no recabados a partir de los descriptores.

La inclusión de los fallos en el corpus se realizó luego de la lectura del resumen, o de la lectura completa en caso de que la información suministrada fuera muy escasa o dudosa. Como criterio de inclusión se definió que los fallos trataran acerca del rechazo de tratamientos, ya sea en situaciones de terminalidad o cuando la omisión o el rechazo de los tratamientos pusieran en riesgo la vida de los pacientes. Los fallos que no se ajustaban a estas situaciones fueron excluidos.

El corpus quedó conformado por 38 fallos. El texto de las sentencias fue codificado y analizado a partir de técnicas cualitativas en el programa ATLAS.ti., siguiendo el esquema de análisis propuesto por Gloppen ${ }^{(31)}$ : en primer lugar, se analizaron las características del reclamo (quiénes y qué demandaban) y, en segundo lugar, las características de la decisión judicial (cuál fue la decisión y con qué argumentos se justificó). Para el análisis descriptivo del corpus se elaboró una matriz con datos del fallo, de la demanda y los demandantes (quién lo originaba, la condición o patología implicada, la orientación y la base jurídica de la demanda) y de la decisión judicial (la sentencia y la base jurídica de la sentencia), entre otros. Ambos abordajes contribuyeron a profundizar la comprensión del fenómeno.

Relevar un corpus exhaustivo de sentencias sobre la temática en un periodo tan amplio es complejo debido a las limitaciones 
temporales de algunos buscadores y al hecho de que no todos los fallos figuran en las bases. Pese a las limitaciones, el conjunto de documentos que se discuten permiten caracterizar los procesos de judicialización del rechazo de tratamientos más allá de los fallos paradigmáticos, a los que se remiten los trabajos que analizan la jurisprudencia desde una perspectiva jurídica o bioética ${ }^{(32,33,34,35)}$.

\section{RESULTADOS}

\section{El derecho a rechazar tratamientos en los tribunales argentinos}

¿Qué características tiene la judicialización en torno al rechazo de tratamientos en Argentina? ¿Qué tipos de casos llegan a los tribunales y cuáles son los motivos por los que se recurre a una instancia de decisión judicial? ¿Quiénes presentan estas demandas y qué respuesta encuentran en los tribunales?

Un primer aspecto a señalar es el bajo grado de judicialización en torno al rechazo de tratamientos en el final de la vida. Al margen de posibles omisiones en el relevamiento, tanto por inconsistencias de las bases como por características propias de estos $\operatorname{casos}^{[a]}$, la cantidad de fallos producidos por tribunales argentinos es muy baja. Se identificaron un total de 38 fallos para el periodo analizado, y si bien el número se incrementó a partir de la década de 1990 no se produjeron oscilaciones significativas durante el periodo. En los últimos años, en los que el tema adquirió mayor visibilidad a raíz de los debates públicos y la sanción de la Ley $26742^{(3)}$, tampoco se evidenció un aumento o descenso notorio respecto de años anteriores. En comparación con la magnitud que ha adquirido la judicialización en temáticas relativas a la salud en las últimas décadas, especialmente reclamos por cobertura de tratamientos ${ }^{(24,25,26)}$, la escasa cantidad de fallos da cuenta del bajo grado de litigación en torno a este derecho.

¿Quiénes acuden al ámbito judicial para resolver disputas en la toma de decisiones médicas en el final de la vida? Los casos judicializados que llegan a sentencia son iniciados mayoritariamente por profesionales médicos o instituciones de salud, como hospitales públicos y clínicas privadas y, en menor medida, por familiares de pacientes (padres, hijos y cónyuges). Solo tres fallos fueron movilizados por pacientes que recurrieron a instancias judiciales, ya sea para dirimir disputas en la toma de decisiones médicas o para garantizar que se respeten sus voluntades.

En Argentina, independientemente de quién lleve el caso a la justicia, los motivos por los que se judicializan este tipo de decisiones tiene un sesgo pro-tratamiento. La mayoría de las sentencias se producen en respuesta a pedidos de autorización para la realización o continuación de intervenciones que son rechazadas por los pacientes, ya sea transfusiones de sangre o procedimientos quirúrgicos.

De las presentaciones iniciadas por profesionales e instituciones de salud, la mayoría son demandas de autorización a la justicia para la realización de tratamientos que son rechazados por pacientes o por sus allegados. Un ejemplo de este tipo de casos puede hallarse en un fallo muy citado en la jurisprudencia, producido por el juez Pedro Hooft, en el que se rechaza el pedido de autorización de una institución de salud para realizar una amputación a un paciente diabético que se niega a ser sometido a dicha operación ${ }^{(36)}$.

Los pedidos intercedidos por familiares de pacientes se dividen entre aquellos que demandan que se retire o que no se inicie un tratamiento -en casos en que los propios pacientes no pueden ejercer su voluntad- $y$ aquellos que se oponen a la decisión autónoma de los pacientes de rechazar tratamientos y reclaman a la justicia que dichas intervenciones se realicen en forma compulsiva. Como ejemplo del primer tipo de presentaciones puede señalarse, entre otros, el caso al que alude el último fallo de la Corte Suprema sobre el tema, en que las hermanas de un paciente en estado vegetativo demandaban el retiro de medidas de soporte vital ${ }^{(1)}$. El pedido de uno de los hijos de un paciente con una enfermedad crónica avanzada, que demanda la realización compulsiva de una amputación quirúrgica que su 
padre rechaza ${ }^{(37)}$, ejemplifica el segundo tipo de presentaciones.

Las escasas demandas presentadas por los pacientes apuntan a garantizar sus decisiones de limitar intervenciones, ya sea en forma preventiva o ante la negativa de los profesionales o instituciones de salud de respetar dichas decisiones. El primer pronunciamiento de la Corte Suprema de Justicia sobre el rechazo de tratamientos -el caso Bahamondez- se inicia por la demanda de un paciente para que se respete su negativa a recibir transfusiones de sangre basada en motivos religiosos $^{(38)}$.

En la jurisprudencia consultada no se identificaron casos en que familiares o pacientes demandaran a la justicia la realización de tratamientos luego de que los profesionales hubieran dispuesto discontinuar o no realizar intervenciones.

Respecto a los tópicos que motivan la judicialización de decisiones médicas en el final de la vida, la gran mayoría obedece a disputas en torno al rechazo de transfusiones sanguíneas por parte de testigos de Jehová, grupo que ha generado una importante jurisprudencia sobre la temática en muchos países. Este tipo de casos fueron los primeros en Ilegar a tribunales y motivaron varios pronunciamientos de la Corte Suprema, como el fallo en el caso Bahamondez, ya citado.

En menor medida, el recurso a los tribunales se inicia por el rechazo de intervenciones quirúrgicas, como la negativa a realizarse amputaciones en patologías crónicas avanzadas como la diabetes, o la oposición al uso de tecnologías que prolongan artificialmente la vida (desde respiradores mecánicos hasta medidas de hidratación y alimentación artificial). Entre estos últimos casos, que comienzan a judicializarse recién a partir de la década de 2000, se distinguen aquellos en que los familiares demandan la suspensión de medidas en curso y quienes acuden a la justicia en forma preventiva, para evitar su uso en el futuro. El pedido de suspensión de la hidratación y alimentación artificial de una mujer en estado vegetativo, realizada por su esposo $^{(39)}$, y la demanda de una mujer que padece una enfermedad degenerativa para que se respete en el futuro su oposición al uso de intervenciones invasivas ${ }^{(40)}$, ilustran estas variaciones.

La mayoría de los casos Ilegan a tribunales cuando los pacientes están en estado crítico (por un accidente o por el avance de la enfermedad) o en la etapa terminal de una enfermedad crónica o degenerativa. En esa instancia, los pedidos apuntan en forma directa a permitir que una muerte inminente ocurra por el retiro del soporte vital o la no realización de una intervención urgente. En menor medida, el recurso a tribunales se produce en situaciones en que el final de la vida no es inminente, como la demanda de los padres de un menor, afectado por una enfermedad degenerativa, para que no se realicen tratamientos agresivos en caso de que en el futuro sobreviniera una crisis que pusiese en riesgo su vida ${ }^{(41)}$.

Cabe mencionar que ninguno de los casos que llegan a tribunales remite a intervenciones relacionadas con la eutanasia o el suicidio asistido, prácticas penalizadas por la legislación argentina como, por ejemplo, pedidos de autorización de acciones directas que tuvieran por objetivo provocar la muerte de un paciente (por ejemplo, las Ilamadas "inyecciones letales"), como sí ocurre en casos judicializados en otros países ${ }^{(42)}$.

Un aspecto relevante de la judicialización en torno al rechazo de tratamientos es que muchos de los fallos del periodo analizado se originaron porque una de las partes recurrió en forma preventiva a los tribunales. Estos pedidos generalmente son cursados por médicos o instituciones de salud, para evitar futuros reclamos judiciales, o por los propios pacientes, para no verse expuestos a tratamientos no deseados en el futuro. La búsqueda de una autorización judicial para medidas que no la requieren de acuerdo a la legislación vigente -como el pedido ya comentado de una mujer en pos de la abstención futura de medidas invasivas ${ }^{(40)}-$, incluso en casos en los que no existe una disputa entre los deseos del paciente, la familia y los profesionales médicos, caracteriza este tipo de demandas. El caso paradigmático de estos pedidos, que expresan el carácter 
"preventivo" de buena parte de la judicialización, es el recurso presentado por una clínica privada para que la justicia autorice que no se transfunda a un paciente testigo de Jehová ${ }^{(43)}$. El fragmento del fallo de la Corte Suprema de Justicia sobre este caso ilustra este punto:

En efecto, los propios términos de dicha petición, al incluir un pedido de autorización judicial para no efectuar transfusiones de sangre al paciente, demuestran, con toda claridad, la inexistencia de conflicto de intereses entre $Z$. y el establecimiento médico. En consecuencia, falta en autos uno de los requisitos fundamentales para autorizar el ejercicio de la potestad judicial, tal es la existencia de "controversias entre partes con intereses jurídicos contrapuestos y propios para la dilucidación jurisdiccional". ${ }^{(43)}$

La sanción de normas o la producción de jurisprudencia relevante en la temática (como pronunciamientos de la Corte Suprema de Justicia) no necesariamente impide la judicialización futura de casos similares, como lo evidencia la litigación luego de la aprobación de la ley de "muerte digna" en 2012, o la presencia recurrente de demandas en torno al rechazo de transfusiones en el periodo analizado, luego de varios fallos del máximo tribunal amparando ese derecho.

Hasta aquí se presentaron datos relativos al perfil de los reclamos o demandas. Ahora bien: ¿cuáles fueron las respuestas de los tribunales a estos pedidos? ¿En qué medida y en qué circunstancias respaldaron el rechazo de tratamientos o intervenciones?

La mayoría de las sentencias relevadas garantizaron el derecho a rechazar tratamientos médicos en el final de la vida. El derecho a rechazar tratamientos se avala por tres vías: legitimando la negativa a tratarse, reclamada por pacientes o sus familiares ${ }^{(40)}$; respaldando pedidos de autorización para no tratar interpuestos por profesionales o instituciones de salud ${ }^{(43)}$; o bien rechazando pedidos de autorización para la realización de intervenciones no deseadas por los pacientes, presentadas tanto por profesionales e instituciones de salud ${ }^{(36)}$ como por familiares de pacientes $^{(37)}$. En los casos que involucran a menores, como los pedidos originados por el rechazo de padres a transfundir a sus hijos ${ }^{(44)}$, las sentencias que avalan el rechazo de tratamientos son minoritarias.

La jurisprudencia que avala el rechazo de tratamientos se afirma en la década de 1990, en la que se producen los primeros fallos de rango constitucional que reconocen la preeminencia de la autonomía de los pacientes en las decisiones respecto a su propio cuerpo $^{(38)}$, y comienza a perfilarse la formulación de un derecho a una muerte digna ${ }^{(36)}$. Las decisiones contrarias a los pedidos de rechazo de tratamientos están sustentadas en la protección de la vida como bien supremo. La base jurídica de las decisiones que respaldan el rechazo de tratamientos, por su parte, varían durante el periodo ${ }^{(30)}$, pero en líneas generales se apoyan en normas constitucionales que garantizan el derecho a la privacidad y la autonomía (los artículos 14 y 19 de la Constitución Nacional Argentina); en la legislación en materia de práctica médica y derecho de los pacientes, como la Ley 17132 de Ejercicio de la Medicina ${ }^{(45)}$, que obliga a los profesionales a respetar la negativa de los pacientes a tratarse; y, luego de 2012, en la Ley $26742^{(2)}$, que modificó la Ley de Derechos de los Pacientes sancionada algunos años antes, con referencias más explícitas al rechazo de tratamientos en el final de la vida.

\section{Justiciabilidad de las decisiones en el final de la vida}

El incremento de la judicialización de temáticas de salud en Argentina ha sido asociado a cambios normativos y procesales que promovieron la litigación en este campo, como el otorgamiento de rango constitucional a tratados que reconocen en forma explícita el derecho a la salud, la incorporación y flexibilización de herramientas que facilitan el acceso a la justicia y el desarrollo de estructuras de apoyo a la judicialización, como abogados especializados en 
la litigación sobre determinadas materias ligadas a la salud ${ }^{(18,24,26)}$. Ahora bien, ¿cuáles son las condiciones de justiciabilidad del derecho a rechazar tratamientos en el final de la vida? ¿Qué factores inciden en la baja justiciabilidad de este derecho?

Entre los elementos que pueden facilitar u obstaculizar los procesos de judicialización en torno a una temática puntual pueden identificarse factores propios del campo legal, como la existencia o no de normativas y estructuras que permitan la litigación, y aquellos que exceden este campo, como procesos sociales y políticos que contribuyan a configurar determinada problemática desde una perspectiva de derechos.

El marco normativo en que se apoyan las demandas ligadas al rechazo de tratamientos ha variado en el periodo analizado. Previamente a la llamada ley de "muerte digna", sancionada en 2012, no existía un marco legal explícito para las decisiones que involucraran la abstención o suspensión de tratamientos que culminaran en la muerte del paciente. Dicha ley aportó claridad respecto al alcance de los derechos de los pacientes terminales, describiendo expresamente las condiciones y los tratamientos pasibles de ser rechazados, con la intención de evitar judicializar las decisiones de los pacientes respecto a la continuación o abstención de tratamientos. Si bien en el marco normativo argentino el derecho a rechazar tratamientos estaba contemplado desde 1967 en la Ley 17132 de Ejercicio de la Medicina(45), que obligaba a los profesionales de la salud a respetar la voluntad de los pacientes cuando se negaran a realizar un tratamiento, diversos actores denunciaban el vacío legal en diferentes instancias y situaciones. La poca claridad de la legislación en el periodo analizado, tanto respecto a qué tratamientos era posible rechazar como a quiénes y en qué circunstancias estaban en condición de hacerlo, posiblemente incidió en el bajo recurso a la justicia por parte de pacientes y familiares. Asimismo, en un contexto marcado por la medicina defensiva y el temor a los juicios por mala praxisis(bib], esta situación contribuye a la judicialización preventiva, movilizada por profesionales e instituciones que buscan cubrirse ante posibles demandas.

La existencia y disponibilidad de estructuras de apoyo legal en la litigación sobre estas temáticas, como podrían serlo abogados y ONG especializados en temas de bioética, también juegan un papel en la justiciabilidad de estos derechos. A diferencia de lo que ocurre en otros países, en Argentina no existen asociaciones destinadas a promover los derechos de los pacientes terminales, y el desarrollo de estructuras de apoyo legal en estos temas es incipiente. La preeminencia de fallos motivados por el rechazo de transfusiones sanguíneas por parte de testigos de Jehová se debe, en parte, a la mayor presencia de apoyos legales en este grupo, ya sea para accionar judicialmente en caso de que las decisiones de sus miembros se vean vulneradas, como en la asistencia para formular voluntades anticipadas. Si en torno a otras condiciones de salud se han generado grupos de pacientes o familiares que se han movilizado políticamente por el reconocimiento de derechos (el VIH/sida es el caso paradigmático), no hubo en Argentina, hasta el año 2011, ningún tipo de movilización que nucleara o representara a pacientes terminales ${ }^{(3)}$.

Otro factor a tener en cuenta es lo que los analistas del derecho Ilaman la estructura de oportunidad jurídica de los posibles litigantes, es decir, el hecho de que los tribunales sean percibidos como el ámbito adecuado y más operativo para dirimir estas disputas $^{(48)}$. En ese sentido, los a menudo prolongados tiempos de los procesos judiciales en el país parecen poco compatibles con los tiempos más acotados en los que estas decisiones tienen lugar. Como hemos visto, la mayoría de las presentaciones judiciales analizadas se producen cuando los pacientes están en periodos críticos (a raíz de accidentes o en ocasión de cirugías) o en estados muy avanzados de una enfermedad progresiva, en los que el tiempo es un recurso escaso. En este marco, el camino judicial puede verse como una vía poco operativa para la resolución de situaciones problemáticas en el final de la vida. 
Uno de los factores que incide en la judicialización de una temática, ya por fuera del ámbito propio de la justicia, es una construcción más amplia de esta como un asunto de derechos ${ }^{(49)}$. Es decir, el grado en el que la ciudadanía percibe, en términos de derechos, la toma de decisiones en situaciones extremas en las que está en juego la dignidad personal, e identifica al Estado como garante del ejercicio pleno de la autonomía en esas instancias. Al respecto, el discurso de los derechos de los pacientes y la centralidad de la autonomía en el campo de la salud es relativamente reciente en países como Argentina, donde han primado el paternalismo y mecanismos informales de confianza en la autoridad médica frente a este tipo de decisiones ${ }^{(6)}$.

En Argentina, las demandas públicas de pacientes y familiares por la sanción de una ley de "muerte digna" en el año 2011 abrieron vías de resolución política a los conflictos en torno a la gestión médica del final de la vida y pusieron en la agenda pública la discusión sobre los derechos de los pacientes sobre su propio cuerpo ${ }^{(3)}$. Previamente a esta coyuntura, la existencia de debates sobre la toma de decisiones en el final de la vida a nivel local fue casi nula, y los fallos judiciales comentados en el apartado anterior no tuvieron la cobertura mediática que sí tuvieron casos similares en otros países, lo que probablemente haya obstaculizado que el rechazo de tratamientos se configure como un tema de derechos, o que la justicia se posicione como un espacio para garantizar la autonomía en estas decisiones.

\section{DISCUSIÓN}

En las líneas anteriores se perfiló la judicialización de decisiones en torno al rechazo de tratamientos en el final de la vida en Argentina y los factores que afectan su justiciabilidad. Dicho proceso está marcado por: 1) el bajo grado de judicialización de casos de rechazo de tratamientos en el final de la vida; 2) la baja proporción de casos presentados por pacientes y familiares, siendo los profesionales e instituciones de salud quienes mayoritariamente movilizan la intervención de la justicia; 3) el sesgo marcadamente protratamiento en las demandas, iniciadas más en pos de autorizar intervenciones contrarias a las preferencias de los pacientes que en virtud del respeto del derecho a rechazar tratamientos; y 4) un alto porcentaje de demandas presentadas en forma preventiva o innecesaria, por la ausencia de conflictos entre los actores involucrados (profesionales, pacientes y familiares) o, en muchos casos, en situaciones que, por las leyes vigentes y la jurisprudencia, no requieren intervención judicial. Entre los factores asociados a la escasa litigación en torno a este derecho se identificaron la falta de precisiones en el marco normativo respecto a las decisiones médicas en el final de la vida, la ausencia de estructuras de apoyo legal y de asociaciones que defiendan los derechos de los pacientes terminales, y la baja percepción del rechazo de tratamientos como un asunto de derechos por parte de la ciudadanía.

Comparar el perfil de la judicialización en este campo con el de otros países resulta complejo dado que, en líneas generales, los estudios que revisan la jurisprudencia sobre la temática se centran más en el análisis de las decisiones judiciales desde un punto de vista legal y/o normativo (si la muerte digna puede considerarse un derecho y en base a qué fundamentos), que en las demandas que las originan y las dinámicas por las cuales los casos Ilegan a los tribunales ${ }^{(32,33,34,35,50,51,52,53)}$. Por ejemplo, revisiones de la jurisprudencia estadounidense dan cuenta de los diferentes tópicos sobre los que han fallado los tribunales, y ponen énfasis en la base jurídica de las sentencias, en temas como el retiro de soporte vital, directivas anticipadas, cuidados paliativos y suicidio asistido ${ }^{(51,52)}$, pero no caracterizan la litigación en torno a estos temas ni permiten inferir tendencias. La bibliografía que examina la sanción de leyes sobre la temática, como la inclusión del rechazo de tratamientos en el marco jurídico de México ${ }^{(54)} y$ Andalucía $^{(55)}$, o la regulación de las directivas anticipadas en Uruguay(56), comentan los alcances y limitaciones de estas normas. 
De todas formas, tanto estos trabajos como los estudios sociológicos que analizan los movimientos que reivindican el derecho a la muerte digna en diferentes países ${ }^{(57,58,59,60)}$ aportan claves para poner en perspectiva el proceso de judicialización de las decisiones médicas en el final de la vida en Argentina.

Un aspecto referido en la bibliografía es el impacto que tiene -tanto en la práctica médica como en la justiciabilidad del rechazo de tratamientos- la falta de claridad en los marcos normativos. La falta de legislación específica, o la interpretación contradictoria de algunas normas, genera incertidumbre respecto a quiénes pueden rechazar tratamientos, qué tratamientos y en qué condiciones. Incluso en países que han incluido en forma explícita el derecho a rechazar tratamientos en el final de la vida, como México, a través de la Ley General de Salud en Materia de Cuidados Paliativos, se carece de precisiones respecto a los tratamientos que pueden ser suspendidos ${ }^{(54)}$. El caso de Colombia es paradigmático al respecto dado que, en dicho país, la Corte Constitucional despenalizó en 1997 algunos casos de eutanasia, permitiendo que un paciente terminal pueda recibir asistencia de un médico para finalizar su vida ${ }^{(54)}$. La no regulación de esta práctica por medio de una ley generó incertidumbre sobre su estatuto legal y esto es lo que buscan remediar las iniciativas actuales, dado que no existían criterios claros acerca de las condiciones en las que esta práctica podía realizarse. En el caso Argentino, la ley sancionada en el año 2012 buscó saldar los puntos ciegos presentes en la legislación previa, explicitando las intervenciones pasibles de ser rechazadas, los sujetos alcanzados y los procedimientos para hacerlo.

Otro aspecto que aparece problematizado en los estudios es el rol que tienen las estructuras de apoyo legal, como abogados especializados en la temática u organizaciones o grupos que respalden estos reclamos, en la litigación en el final de la vida. La presencia o ausencia de dichas estructuras, y más precisamente de organizaciones que defiendan los derechos de los pacientes terminales, impacta en las condiciones para judicializar las decisiones médicas en el final de la vida. En Argentina, como en la mayoría de los países de la región, a excepción de Colombia ${ }^{[c]}$, no existen organizaciones de este tipo, a diferencia de lo que ocurre en algunos países anglosajones. La judicialización de demandas presentadas por pacientes terminales o familiares de pacientes en países como EE.UU. y Reino Unido suelen estar impulsadas y patrocinadas por grupos de activistas nucleados en asociaciones destinadas a promover el reconocimiento de derechos en el final de la vida, que apelan a la judicialización y a los casos de alto impacto como estrategia para visibilizar sus reclamos ${ }^{(42,58,59,60)}$. En las últimas décadas, los debates sobre el derecho a morir en el Reino Unido han girado en torno a procesos judiciales de alto perfil público, y organizaciones como Dignity in dying, que abogan por la legalización de la eutanasia y el suicidio asistido, brindan asesoramiento jurídico a personas que buscan acceder a una muerte digna ${ }^{(42,58)[d]}$. Por su parte, la organización Compassion in dying, que impulsó reformas a través de referéndums para legalizar el suicidio asistido en varios estados de EE.UU. (como Oregon, primer estado en legalizar estas prácticas), paralelamente recurrió a los tribunales para conseguir sus objetivos ${ }^{(60)}$. A través de demandas encabezadas conjuntamente por pacientes y profesionales, cuestionaron la constitucionalidad de normativas que prohibían en forma explícita el suicidio asistido en algunos estados, pero los reclamos fueron rechazados por la Corte Suprema ${ }^{(60)}$.

Este tipo de patrocinio influye en los procesos de litigación al menos de dos formas: por un lado, en la manifestación de una litigación estratégica, destinada a obtener no solo respuestas individuales sino el reconocimiento de los derechos de los pacientes ${ }^{(42,57)}$. Por otro, la presencia de estas estructuras incide en la radicalidad de las demandas. En Argentina, no hay antecedentes de demandas de autorización de prácticas asociadas a la eutanasia o el suicidio asistido, como en los casos comentados.

Por último, el grado de visibilidad de los reclamos y debates en torno a las decisiones 
en el final de la vida afecta al reconocimiento de derechos por parte de la ciudadanía. En América Latina estas temáticas no se han configurado como tópico de discusión pública sino de forma excepcional[e]. Cuestiones como la eutanasia, el suicidio asistido y los límites en el uso del soporte vital no han ingresado en la agenda política con la fuerza en que lo han hecho, en mayor o menor medida, otros tópicos sensibles como el aborto. En Argentina, previo a las demandas de pacientes y familiares por la sanción de una ley de "muerte digna" en el año 2011, que evitaron ex profeso la vía judicial para canalizar sus reclamos, la temática estuvo prácticamente ausente en la agenda pública ${ }^{(3)}$. Dilucidar el impacto de la visibilidad de estos dramas privados en las prácticas de profesionales y pacientes, así como la politización de las demandas en la apropiación de derechos en el final de la vida por parte de la ciudadanía, deberá evaluarse en futuras indagaciones.

\section{CONCLUSIONES}

En este artículo se describió la judicialización del rechazo de tratamientos en el final de la vida en los tribunales argentinos, y se discutieron las condiciones para la justiciabilidad de este derecho. A partir del análisis de un conjunto de fallos judiciales producidos a lo largo de cuatro décadas (1975-2015), se describió el perfil de los litigantes y de los casos judicializados, así como la orientación de las sentencias. Asimismo se abordaron factores judiciales y extrajudiciales que podrían dar cuenta de la baja justiciabilidad de las decisiones de retirar o suspender tratamientos médicos en situaciones críticas en las que está en juego el final de la vida.

Algunos trabajos que analizan el avance de la judicialización en el campo de la salud señalan la importancia de estos recursos para garantizar el cumplimiento de derechos, mientras que otros advierten algunas consecuencias negativas ${ }^{(21,22,24)}$, entre las que se menciona el establecimiento de una medicina defensiva ${ }^{(6)}$. La intromisión innecesaria de tribunales y jueces en la toma de decisiones médicas implicaría una amenaza para la autoridad de los profesionales de la salud en un dominio antes percibido como no problemático.

El bajo grado de judicialización de este tipo de decisiones, como el protagonismo que tienen los propios profesionales médicos y el sesgo pro-tratamiento de las demandas en esta materia, que buscan ya no garantizar sino limitar el poder de decisión de los pacientes, obligan a ser cautos respecto a las implicancias de los procesos de judicialización en las decisiones médicas en el final de la vida, así como a considerar en qué medida estos suponen un mayor empoderamiento de los pacientes respecto a sus derechos o una mayor conflictividad en la relación médicopaciente, como podría pensarse a priori.

A la luz de los cambios recientes en la legislación nacional, y la configuración de las decisiones en el final de la vida como un tópico de discusión pública en el país ${ }^{(3,4,5)}$, cabe plantear algunos interrogantes sobre las derivas futuras de la judicialización en este campo: ¿en qué medida la mayor precisión de la normativa supondrá una menor presencia de este tipo de casos en los tribunales $\mathrm{o}$, contrariamente y como ha ocurrido con el reconocimiento constitucional del derecho a la salud, explicitar y clarificar estos derechos aumentará las acciones judiciales? ¿Una mayor visibilización y politización de las decisiones en el final de la vida contribuirá a empoderar a los pacientes para reclamar por sus derechos o facilitará decisiones consensuadas con los profesionales médicos? ¿En qué medida la nueva legislación cambiará las prácticas de los profesionales y permitirá garantizar el respeto de los derechos de los pacientes?

Evaluar adecuadamente el impacto que los cambios en la visibilidad de la temática y el marco jurídico tendrán en la judicialización de la toma de decisiones al final de la vida en los años próximos, así como en los efectos de estos procesos para la resolución de conflictos en contextos médicos (entre pacientes, familiares y profesionales de la salud), requerirá de estudios que permitan 
reconstruir las diferentes aristas del problema en toda su complejidad. Observar la evolución de la jurisprudencia sobre la temática en años próximos, y examinar las actitudes y prácticas de profesionales de la salud y de la ciudadanía respecto a las decisiones médicas en el final de la vida permitirán ponderar el verdadero impacto de estos procesos. Finalmente, comparar la judicialización del rechazo de tratamientos en países con diferentes marcos normativos y con experiencias disímiles en la politización de reclamos relativos a la dignidad en el final de la vida contribuirá a un mejor entendimiento de los factores involucrados en la configuración de la muerte digna como un asunto de derechos.

\section{AGRADECIMIENTOS}

La investigación "Construyendo una 'muerte digna': discursos y prácticas en torno a la gestión médica del final de la vida en Argentina", que da origen a este artículo, fue financiada por el Consejo Nacional de Investigaciones Científicas y Técnicas (CONICET) y por un subsidio de la Agencia Nacional de Promoción Científica y Tecnológica (PICT 2012-0938). Los comentarios de los evaluadores anónimos permitieron mejorar en forma significativa el artículo.

\section{REFERENCIAS BIBLIOGRÁFICAS}

1. Corte Suprema de Justicia de la Nación. D.M.A s/declaración de incapacidad, CSJ 376/2013 (49-D) CS1 [Internet]. Buenos Aires: 7 jul 2015 [citado 12 mar 2016]. Disponible en: http://goo.gl/SdXBBB.

2. Honorable Cámara de Diputados de la Nación Argentina. Ley 26742: Modifícase la Ley $\mathrm{N}^{\circ}$ 26.529 que estableció los derechos del paciente en su relación con los profesionales e instituciones de la Salud [Internet]. Buenos Aires: InfoLEG Información Legislativa; 2012 [citado 10 abril 2015]. Disponible en: http://goo.gl/6sfUI0.

3. Alonso JP, Luxardo N, Poy S, Bigalli M. El final de la vida como objeto de debate público: avatares de la "muerte digna" en Argentina. Sociedad. 2014;33:7-20.

4. Alonso JP. De medios ordinarios y extraordinarios. La Iglesia Católica y los debates sobre la "muerte digna" en Argentina. Religião e Sociedade. 2014;34(1):122-145.

5. Irrazábal G. Religión y salud: la intervención pública de agentes religiosos católicos formados en bioética en el debate parlamentario sobre la muerte digna en Argentina. Salud Colectiva. 2015;11(3):331-349.
6. Gherardi C, Gherardi N. La judicialización del acto médico y la generación de nuevos conflictos. Medicina (Buenos Aires). 2007;67:502-510.

7. Rothman D. Strangers at the bedside: a history of how law and bioethics transformed medical decision making. New York: Basic Books; 1991.

8. Ariès P. Morir en Occidente: desde la Edad Media hasta la actualidad. Buenos Aires: Adriana Hidalgo; 2000.

9. Illich I. Némesis médica: La expropiación de la salud. México: Joaquín Mortiz; 1978.

10. Lock M. Twice dead: organ transplants and the reinvention of death. Berkeley: University of California Press; 2002.

11. Kaufman S. And a time to die: how American hospitals shape the end of life. Chicago: University of Chicago Press; 2006.

12. Abdo Ferez C. La política y la juristocracia. Sociedad. 2014;33:51-72.

13. Asensi F. Judicialização ou juridicização? As instituições jurídicas e suas estratégias na saúde. Physis. 2010;20(1):33-55.

14. Carvalho ER. Em busca da judicialização da política no Brasil: apontamentos para uma nova abordagem. Revista Sociologia Política. 2004;(23):115-126.

15. Cuenca A, Piccone MV. Nuevos movimientos sociales en Argentina y judicialización de demandas. Derecho y Ciencias Sociales. 2011;(5):30-50.

16. Delamata G. Amanecer y progreso de un repertorio social activista de derechos bajo la democracia recuperada. Revista SAAP. 2013;7(2):307-315.

17. Sieder R, Schjolden L, Angall A, (orgs). La judicialización de la política en América Latina. Bogotá: Universidad Externado de Colombia; 2008. 
18. Smulovitz C. Petición y creación de derechos: la judicialización en Argentina. En: Sieder R, Schjolden L, Angall A, (orgs). La judicialización de la política en América Latina. Bogotá: Universidad Externado de Colombia; 2008. p. 193-221.

19. Smulovitz C. Organizaciones que invocan derechos: Sociedad civil y representación en Argentina. POSTData. 2009;(13):51-79.

20. Ventura M. A transexualidade no tribunal: saúde e cidadania. Río de Janeiro: EdUERJ; 2010.

21. Motta Ferraz OL. The right to health in the courts of Brazil: worsening health inequities? Health and Human Rights. 2009;11(2):33-45.

22. Motta Ferraz OL. Brasil: desigualdades en salud, derechos y tribunales: El impacto social de la judicialización de la salud. En: Yamín A, Gloppen S, (orgs). La lucha por los derechos de la salud. Buenos Aires: Siglo XXI Editores; 2013. p. 97-126.

23. Ventura M, Simas L, Pepe V, Schramm F. Judicialização da saúde, acesso à justiça e efetividade do direito a saúde. Physis. 2010;20(1):77-100.

24. Bergallo P. Argentina: los tribunales y el derecho a la salud. ¿Se logra justicia a pesar de la "rutinización" de los reclamos individuales de cobertura? En: Yamín A, Gloppen S, (orgs). La lucha por los derechos de la salud. Buenos Aires: Siglo XXI Editores; 2013. p. 59-95.

25. Yamín A, Parra-Vera O, Gianella C. Colombia: la protección judicial del derecho a la salud: ¿Una promesa difícil de cumplir? En: Yamín A, Gloppen S, (orgs). La lucha por los derechos de la salud. Buenos Aires: Siglo XXI Editores; 2013. p. 127-158.

26. Abramovich $\mathrm{V}$, Pautassi L. El derecho a la salud en los tribunales: Algunos efectos del activismo judicial sobre el sistema de salud en Argentina. Salud Colectiva. 2008;4(3):261-282.

27. Diniz D, Machado T, Penalva J. A judicialização da saúde no Distrito Federal, Brasil. Ciência \& Saúde Coletiva. 2014;19(2):591-598.

28. Diniz D. Quem autoriza o aborto seletivo no Brasil?: Médicos, promotores e juízes em cena. Physis. 2003;13(2):251-272.

29. Diniz D. Quando a morte é um ato de cuidado: obstinação terapêutica em crianças. Cadernos de Saúde Pública. 2006;22(8):1741-1748.

30. Alonso JP. El derecho a una muerte digna en Argentina: la judicialización de la toma de deci- siones médicas en el final de la vida. Physis. 2016 26(2):569-589.

31. Gloppen S. La lucha por los derechos de la salud: Marco de análisis. En: Yamín A, Gloppen S, (orgs). La lucha por los derechos de la salud. Buenos Aires: Siglo XXI Editores; 2013. p. 31-43.

32. Hooft P. Bioética y jurisprudencia. Acta Bioethica. 2002;8(2):211-237.

33. Hooft P, Picardi G. Bioética y derecho: El consentimiento informado en la Jurisprudencia Argentina. Revista Latinoamericana de Bioética. 2006;(10):1-58.

34. Magnante D. Tratamientos proporcionados y desproporcionados en el estado vegetativo persistente. Vida y Ética. 2010;11(1):299-345.

35. Urbina PA. El derecho a rechazar prácticas médicas en el final de la vida: Algunas reflexiones a partir de la legislación, doctrina y jurisprudencia argentina. Revista Científica de la UCES. 2010;14(2):94-108.

36. Juzgado de Primera Instancia en lo Criminal y Correccional $\mathrm{N}^{\circ}$ 3. "Dirección del Hospital Interzonal General de Agudos (HIGA) de Mar del Plata s/ Presentación". Mar del Plata: Juzgado Federal de Mar del Plata; 18 sep 1995.

37. Cámara Nacional de Apelaciones en lo Civil. Sala H. "J., J.". Ciudad Autónoma de Buenos Aires: Cámara Nacional de Apelaciones en lo Civil; 21 feb 1991.

38. Corte Suprema de Justicia de la Nación. "Bahamondez, Marcelo s/ medida cautelar, B. 605. XXII T.316 P.479. [Internet]. Buenos Aires: 6 abr 1993 [citado 26 ago 2016]. Disponible en: http:// goo.gl/rKuvy3.

39. Suprema Corte de Justicia de la Provincia de Buenos Aires. "S., M. d. C. Insania". Buenos Aires: Suprema Corte de Justicia de la Provincia de Buenos Aires; 9 feb 2005.

40. Juzgado en lo Criminal y Correccional $N^{0} 1$. "M." Mar del Plata: Juzgado Federal de Mar del Plata; 25 jul 2005.

41. Juzgado en lo Correccional No 4. "M. d. C. M. y N. A. A". Mar del Plata: Juzgado Federal de Mar del Plata; 27 dic 2006.

42. Richards N. Dying to go to court: demanding a legal remedy to end of life. In: Harper, lan, (org). The clinic and the court: Law, medicine and anthropology. Cambridge: Cambridge University Press; 2015. p. 214-238. 
43. Corte Suprema de Justicia de la Nación. "Prestaciones Médico Asistenciales S.A s/ autorización" [Internet]. Buenos Aires: 8 ago 1996. [citado 26 ago 2016]. Disponible en: http://goo.gl/Y9cpne.

44. Juzgado de la Familia y el Menor. "Asesoría de Menores S/ Solicita protección de persona (A.F.)". Santa Rosa: Juzgado de la Familia y el Menor de La Pampa; 17 oct 2012.

45. Presidencia de la Nación Argentina. Ley 17.132 Reglas para el ejercicio de la medicina, odontología y actividades de colaboración de las mismas [Internet]. Buenos Aires: Información Legislativa, Ministerio de Economía; 1967 [citado 10 abr 2015]. Disponible en: http://goo.gl/fBjgoc.

46. Garabeytan E. Crecen las demandas por mala praxis y se hacen más costosas para el sistema. Perfil. 13 oct 2013: Sec. Sociedad: 23.

47. Corte Interamericana de Derechos Humanos. Caso Suárez Peralta vs. Ecuador: sentencia de 21 de mayo de 2013 [Internet]. San José: Corte IDH; 2013 [citado 17 jul 2016]. Disponible en: http:// goo.gl/FFrh3y.

48. Gloppen S, Roseman M. Introducción: ¿Pueden los litigios judiciales volver más justa la salud? En: Yamín A, Gloppen S, (orgs). La lucha por los derechos de la salud. Buenos Aires: Siglo XXI Editores; 2013. p. 13-30.

49. Pecheny M. La construction de I'avortement et du sida en tant que questions politiques. Lille: Presses Universitaires du Septentrion; 2001.

50. Maglio I, Wierzba S, Belli L, Somers ME. El derecho en los finales de la vida y el concepto de muerte digna. Revista Americana de Medicina Respiratoria. 2016;16(1):71-77.

51. Cantor NL. Twenty-five years after Quinlan: a review of the jurisprudence of death and dying. Journal of Law, Medicine \& Ethics. 2001;29:182-196.

52. Gostin L. Deciding life and death in the courtroom from Quinlan to Cruzan, Glucksberg, and Vacco: A brief history and analysis of constitutional protection of the 'Right to Die'. JAMA. 1997;278(18):1523-1528.

53. Guerra Y. Ley, jurisprudencia y eutanasia: introducción al estudio de la normatividad comparada a la luz del caso colombiano. Revista Latinoamericana de Bioética. 2013;13(2):70-85.

54. Álvarez del Río A. Algunos avances en la regulación sobre la eutanasia en América Latina: el caso de Colombia y México. Perspectivas Bioéticas. 2009;14(26-27):162-167.
55. Bonilla Sánchez JJ. El derecho a morir dignamente en Andalucía. Espacio y Tiempo. 2011;25:171-188.

56. Benitez R. Las voluntades anticipadas en Uruguay: reflexiones sobre la Ley 18473 . Revista IUS. 2015;9(36):135-154.

57. Mclnerney F. "Request death": a new social movement. Social Science \& Medicine. 2000;50(1): 137-154.

58. Richards N. The death of right-to-die campaigners. Anthropology Today. 2014;30(3):14-17.

59. Richards N. The fight-to-die: older people and death activism. International Journal of Ageing and Later Life. 2012;7(1):7-32.

60. Hillyard D, Dombrick J. Dying right. The death with dignity movement. New York: Routledge; 2001.

61. The World Federation of Right to Die Societies. Member Organizations [Internet]. 2016 [citado 12 may 2016]. Disponible en: http://goo.gl/RB4Gte.

62. Menezes RA, Ventura M. Ortotanásia, sofrimento e dignidade: Entre valores morais, medicina e direito. Revista Brasileira de Ciências Sociais. 2013;28(81):213-229.

\section{NOTAS FINALES}

[a] Los procesos que se inician y no llegan a instancia de sentencia porque el fallecimiento del paciente ocurre durante el trámite judicial, situación no inusual en este tipo de litigios, dificultan cuantificar con mayor exactitud la magnitud de la judicialización en este campo.

[b] Si bien no hay datos estadísticos precisos, relevamiento privados dan cuenta del aumento de las demandas por mala praxis en el país, a la vez que de la baja proporción de reclamos que prosperan en la justicia ${ }^{(46)}$. Smulovitz ${ }^{(18)}$ señala que esta brecha puede deberse tanto a excesos en la litigación como a la baja predisposición de los juzgados a dar curso a estos reclamos. Al respecto, cabe citar un fallo de la Corte Interamericana de Derechos Humanos que, a raíz de retrasos y omisiones de la justicia de Ecuador frente a un juicio por mala praxis médica, sentó precedentes respecto a la obligación de los estados nacionales de garantizar el derecho a la integridad personal de los ciudadanos frente a casos de este tipo ${ }^{(47)}$.

[c] Colombia es el único país de América latina que integra la World Federation of Right To Die 
Societies, organización que nuclea a grupos que militan por la autodeterminación de las personas en el final de la vida ${ }^{(61)}$.

[d] Tony Nicklinson, afectado por el síndrome de locked-in, y Debbie Purdy, por esclerosis múltiple, reclamaron a la justicia bajo el patrocinio de esta organización para que se les brindara asistencia para terminar con sus vidas. En ambos casos la justicia rechazó los pedidos ${ }^{(42,58)}$. [e] Entre las excepciones, pueden referirse los debates en torno al fallo ya comentado de la Corte de Colombia ${ }^{(54)}$, la sanción de una ley de muerte digna en Uruguay en $2009^{(56)}$, o el proceso judicial por las reformas del Código de Ética Médica de Brasil que permitía rechazar tratamientos innecesarios en situaciones terminales ${ }^{(62)}$.

FORMA DE CITAR

Alonso JP. Derechos en el final de la vida: el rechazo de tratamientos médicos en los tribunales argentinos. Salud Colectiva. 2016;12(3):383-396. doi: 10.18294/sc.2016.989

Recibido: 5 de noviembre de 2015 | Versión final: 17 de junio de 2016 | Aprobado: 30 de junio de 2016

Este obra está bajo una licencia de Creative Commons Reconocimiento-NoComercial 4.0 Internacional. Reconocimiento - Permite copiar, distribuir y comunicar públicamente la obra. A cambio, se debe reconocer y citar al autor original. No Comercial - Esta obra no puede ser utilizada con finalidades comerciales, a menos que se obtenga el permiso.

http://dx.doi.org/10.18294/sc.2016.989 